\title{
Lyapunov-Based Output Feedback Learning Control of Robot Manipulators
}

\author{
K. Merve Dogan, Enver Tatlicioglu ${ }^{\star}$, Erkan Zergeroglu, and Kamil Cetin
}

\begin{abstract}
This paper address the output feedback learning tracking control problem for robot manipulators with repetitive desired joint level trajectories. Specifically, an observer-based output feedback learning controller for periodic trajectories with known period have been proposed. The proposed learning controller guarantees semi-global asymptotic tracking despite the existence of parametric uncertainties associated with the robot dynamics and lack of velocity measurements. A learningbased feedforward term in conjunction with a novel observer formulation is designed to obtain the aforementioned result. The stability of the controller-observer couple is guaranteed via Lyapunov based arguments. Numerical studies performed on a two link robot manipulator are also presented to demonstrate the viability of the proposed method.
\end{abstract}

\section{INTRODUCTION}

The main purpose of using robotic automation in nearly all different fields industry, is to perform repetitious tasks. Therefore robots used in an industrial application mostly perform a predefined task over and over again. Given the nonlinear nature of the robot dynamics, the need to achieve better tracking performance despite system uncertainties and periodic disturbances related to the periodic task, learning controllers among other nonlinear model based controllers are the preferred controller choice. Moreover, compared to other controller formulations, repetitive learning controllers are computationally efficient, can compensate disturbance terms without the need of high frequency or high gain feedback terms and can deal with time-varying disturbances.

Some of the initial work on repetitive learning control research for robotic systems was made by [1], [2], and [3]; where asymptotic convergence of the aforementioned control schemes can only be guaranteed under restrictive conditions on the plant dynamics. Later to enhance the robustness of [1], [2] modified the repetitive update rule to include the so-called Q-filter. In an attempt to increase the robustness of the previously proposed repetitive learning algorithm [4] and [5] proposed a scheme that exploited the use of kernel functions in the update rule. Sadegh et al. in [6] also proposed to enhance the robustness of the repetitive

\footnotetext{
${ }^{\star}$ To whom all the correspondence should be addressed.

This work is funded by The Scientific and Technological Research Council of Turkey via grant number 113E147.

K. M. Dogan, and E. Tatlicioglu are with the Department of Electrical \& Electronics Engineering, Izmir Institute of Technology, 35430, Urla, Izmir, Turkey (Phone: +90 (232) 7506536; Fax: +90 (232) 7506599; Email: [mervedogan,enver]@iyte.edu.tr).

E. Zergeroglu is with the Department of Computer Engineering, Gebze Institute of Technology, 41400, Gebze, Kocaeli, Turkey (Email: ezerger@bilmuh.gyte.edu.tr).

K. Cetin is with the Department of Electrical \& Electronics Engineering, Gediz University, 35665, Menemen, Izmir, Turkey (Email: kamilcetin@gediz.edu.tr).
}

learning controllers by using a saturated update rule. In [7], authors presented a full state feedback learning controller that achieves asymptotic tracking backed up by a Lyapunov based stability analysis.

All of the controllers mentioned above are full state feedback controllers, that is the controller formulation requires both the position and velocity measurements. However nearly all industrial robots only have position sensors. And it is a known fact that using numerical differentiation to numerically form the velocity signal from position information introduces extra noise to the system. Therefore many researchers were also motivated to design output feedback learning controllers that does not require link velocity measurements. To name a few, in [8] and [9] neural network based reinforcement-learning controllers were presented for different classes of nonlinear discrete-time systems. In [10], a learning controller for a class of single-input, singleoutput, minimum phase, nonlinear, time-invariant systems with unknown output-dependent nonlinearities, unknown parameters and known relative degree $\rho$ is considered.

In this study, by making use of a model free observer together with a novel feedforward learning term, we were able to design an output feedback repetitive learning type controller for robotic manipulators with periodic joint level trajectories. The proposed method ensures asymptotic tracking despite the uncertainties associated with the robot dynamics and lack of velocity measurements. Overall stability of the observer-controller couple was ensured via the use of Lyapunov based arguments. The rest of the text is organized in the following manner; The robot dynamics and its properties are given in Section 2; The observer-controller design and closed loop definitions are presented in Section 3. Stability analysis of the overall closed loop system is detailed in Section 4 while the numerical studies performed on a two link planar robot manipulators are presented in Section 5. Concluding remarks are given in Section 6.

\section{System MODEL AND PROPERTIES}

The dynamic model of an $n$ degree of freedom, direct drive robot manipulator is given in the following form [11], [12]

$$
M(q) \ddot{q}+V_{m}(q, \dot{q}) \dot{q}+G(q)+F_{d} \dot{q}=\tau
$$

where $q(t), \dot{q}(t), \ddot{q}(t) \in \mathbb{R}^{n}$ denote the joint positions, velocities, and accelerations, respectively, $M(q) \in \mathbb{R}^{n \times n}$ is the positive-definite and symmetric inertia matrix, $V_{m}(q, \dot{q}) \in$ $\mathbb{R}^{n \times n}$ is the centripetal-Coriolis terms, $G(q) \in \mathbb{R}^{n}$ is the gravitational effects, $F_{d} \in \mathbb{R}^{n}$ is the constant, diagonal, positive-definite, viscous frictional effects, and $\tau(t) \in \mathbb{R}^{n}$ is 
the control torque input. We will assume that the left-hand side of (1) is first-order differentiable. The dynamic model given by (1) satisfies the following properties that will later be utilized in the controller design and the accompanying stability analysis:

Property 1: The inertia matrix $M(q)$ satisfies the following inequalities [13]

$$
m_{1} I_{n} \leq M(q) \leq m_{2} I_{n}
$$

where $m_{1}, m_{2} \in \mathbb{R}$ are known positive-definite bounding constants, $I_{n} \in \mathbb{R}^{n \times n}$ is the standard identity matrix. Likewise the inverse of $M(q)$ can be bounded as

$$
\frac{1}{m_{2}} I_{n} \leq M^{-1}(q) \leq \frac{1}{m_{1}} I_{n}
$$

Property 2: The inertia and centripetal-Coriolis matrices satisfy the following equalities [13]

$$
\xi^{T}\left(\dot{M}-2 V_{m}\right) \xi=0 \forall \xi \in \mathbb{R}^{n} .
$$

Property 3: The centripetal-Coriolis matrix satisfies the following expression [14]

$$
V_{m}(q, \nu) \xi=V_{m}(q, \xi) \nu \forall \nu, \xi, \in \mathbb{R}^{n} .
$$

Property 4: The norm ${ }^{1}$ of centripetal-Coriolis matrix also satisfies the following inequalities

$$
\left\|V_{m}(q, \xi)\right\|_{i \infty} \leq \zeta_{c 1}\|\xi\| \forall \xi \in \mathbb{R}^{n} .
$$

The norm of frictional effects can be upper bounded as follows

$$
\left\|F_{d}\right\|_{i \infty} \leq \zeta_{f}
$$

where $\zeta_{c 1}$ and $\zeta_{f}$ are positive-definite bounding constants.

Property 5: The robot dynamics given in (1) is linearly parameterized as follows

$$
W(q, \dot{q}, \ddot{q})=M(q) \ddot{q}+V_{m}(q, \dot{q}) \dot{q}+G(q)+F_{d} \dot{q}
$$

where $W(q, \dot{q}, \ddot{q}) \in \mathbb{R}^{n}$ is the regression matrix. The above expression (8) is also written in terms of the desired trajectory in the following form

$$
W_{d}\left(q_{d}, \dot{q}_{d}, \ddot{q}_{d}\right)=M\left(q_{d}\right) \ddot{q}_{d}+V_{m}\left(q_{d}, \dot{q}_{d}\right) \dot{q}_{d}+G\left(q_{d}\right)+F_{d} \dot{q}_{d}
$$

where $W_{d}\left(q_{d}, \dot{q}_{d}, \ddot{q}_{d}\right) \in \mathbb{R}^{n}$ is the desired regression matrix and $q_{d}, \dot{q}_{d}, \ddot{q}_{d} \in \mathbb{R}^{n}$ denotes the desired link position, velocity and acceleration, respectively.

Property 6: The dynamic terms in (1) can be upper bounded as follows [14], [6]

$$
\begin{aligned}
\|M(\xi)-M(\nu)\|_{i \infty} & \leq \zeta_{M 1}\|\xi-\nu\| \\
\left\|M^{-1}(\xi)-M^{-1}(\nu)\right\|_{i \infty} & \leq \zeta_{M 2}\|\xi-\nu\| \\
\left\|V_{m}(\xi, w)-V_{m}(\nu, w)\right\|_{i \infty} & \leq \zeta_{c 2}\|\xi-\nu\|\|w\| \\
\|G(\xi)-G(\nu)\|_{i \infty} & \leq \zeta_{g}\|\xi-\nu\|
\end{aligned}
$$

$\forall \xi, \nu, w \in \mathbb{R}^{n}$, where $\zeta_{M 1}, \zeta_{M 1}, \zeta_{C 2}, \zeta_{g} \in \mathbb{R}$ are positivedefinite bounding constants.

\footnotetext{
${ }^{1}$ The matrix norms presented are actually induced infinity norms, however for the ease of the presentation, standard norm representation is used.
}

\section{OBSERVER-CONTROLLER FORMULATION}

As our goal is to design an output feedback controller, we are under the restriction that only the link position $q(t)$ are available for the controller design. To quantify the control objective, we define the link position tracking error, denoted by $e(t) \in \mathbb{R}^{n}$, as

$$
e \triangleq q_{d}-q
$$

where the desired joint space trajectory $q_{d}(t)$ is periodic with its first three time derivatives. That is;

$$
q_{d}(t)=q_{d}(t-T), \dot{q}_{d}(t)=\dot{q}_{d}(t-T), \ddot{q}_{d}(t)=\ddot{q}_{d}(t-T)
$$

where $T$ is a known constant.

The development of the estimate of the unmeasurable link velocity signal is initiated by introducing the velocity observation error signal $\dot{\tilde{q}}(t) \in \mathbb{R}^{n}$ which is defined as

$$
\dot{\tilde{q}} \triangleq \dot{q}-\dot{\hat{q}}
$$

with the auxiliary position observation error $\tilde{q}(t) \in \mathbb{R}^{n}$ defined similarly as

$$
\tilde{q} \triangleq q-\hat{q}
$$

where $\dot{\tilde{q}}(t), \hat{q}(t)$ and $\dot{\hat{q}}(t) \in \mathbb{R}^{n}$ denote velocity observation error, observed joint position and observed joint velocity signals, respectively. At this stage we will define two auxiliary signals, namely a filtered tracking error signal, denoted by $r(t) \in \mathbb{R}^{n}$, and a filtered version of observation error signal, denoted by $s(t) \in \mathbb{R}^{n}$ in order to ease the presentation of the subsequent analysis

$$
\begin{aligned}
& r \triangleq \dot{e}+\alpha e \\
& s \triangleq \dot{\tilde{q}}+\alpha \tilde{q}
\end{aligned}
$$

where $\alpha \in \mathbb{R}$ is a constant, positive-definite, diagonal control gain. Based on the subsequent stability analysis, the velocity observer is designed as

$$
\dot{\hat{q}}=p+k_{0} \tilde{q}-k_{c} e
$$

where $p(t) \in \mathbb{R}^{n}$ is an auxiliary variable with proper initial value and is updated according to

$$
\dot{p}=k_{1} \operatorname{Sgn}(\tilde{q})+k_{2} \tilde{q}-\alpha k_{c} e
$$

with $k_{0}, k_{c}, k_{1}, k_{2} \in \mathbb{R}^{n \times n}$ being positive-definite, diagonal gain matrices, and $\operatorname{Sgn}(\cdot) \in \mathbb{R}^{n}$ is defined as follows

$$
\operatorname{Sgn}(\varsigma)=\left[\operatorname{sgn}\left(\varsigma_{1}\right), \operatorname{sgn}\left(\varsigma_{2}\right), \cdots, \operatorname{sgn}\left(\varsigma_{n}\right)\right]^{T} \forall \varsigma \in \mathbb{R}^{n} .
$$

The control input torque $\tau(t)$ is designed as the following form

$$
\tau=\hat{W}+k_{p} e+k_{c} \alpha\left(q_{d}-\hat{q}\right)+k_{c}\left(\dot{q}_{d}-\dot{\hat{q}}\right)
$$

where $k_{p} \in \mathbb{R}^{n \times n}$ is a positive-definite, diagonal gain matrix with the feedforward learning term $\hat{W}(t) \in \mathbb{R}^{n}$ is updated according to

$$
\hat{W}(t)=\operatorname{Sat}_{\beta}(\hat{W}(t-T))+k_{L} \alpha\left(q_{d}-\hat{q}\right)+k_{L}\left(\dot{q}_{d}-\dot{\hat{q}}\right)
$$


where $k_{L} \in \mathbb{R}$ is a positive-definite constant control gain, $\beta \in \mathbb{R}$ denotes the limits of the vector saturation function $\operatorname{Sat}_{\beta}(\cdot) \in \mathbb{R}^{n}$. We would like to point out that though the observer-controller formulation presented above does not require joint velocity measurement but for the ease of the presentation we would make use of the following property

$$
\begin{aligned}
q_{d}-\hat{q} & =e+\tilde{q} \\
\dot{q}_{d}-\dot{\hat{q}}+\alpha\left(q_{d}-\hat{q}\right) & =r+s
\end{aligned}
$$

so that we can re-arrange the controller of (23) and the learning term (24) in the following form

$$
\begin{aligned}
\tau & =\hat{W}+k_{p} e+k_{c}(r+s) \\
\hat{W}(t) & =\operatorname{Sat}_{\beta}(\hat{W}(t-T))+k_{L}(r+s) .
\end{aligned}
$$

In the rest of the paper, we will make use of (27) and (28) formulations instead of the velocity free version for analysis purposes only.

\section{A. Observer Analysis}

To this end we take the time derivative of (19), insert for (20), (27), (28), cancel out some common terms and selecting the observation gain $k_{0}$ to satisfy

$$
k_{2}=\alpha\left(k_{0}-\alpha I_{n}\right)
$$

so that we would obtain the dynamics of the filtered version of the observation error in the following form

$$
\dot{s}=N_{d}+N_{b}-k_{1} \operatorname{Sgn}(\tilde{q})+k_{c} r-\frac{1}{\alpha} k_{2} s
$$

where the auxiliary signals $N_{d}\left(q, q_{d}, \dot{q}_{d}, \ddot{q}_{d}, t\right) \in \mathbb{R}^{n}$ and $N_{b}\left(q, \dot{q}, q_{d}, \dot{q}_{d}, e, r, s, t\right) \in \mathbb{R}^{n}$ are defined as

$$
N_{d} \triangleq \ddot{q}_{d}+M^{-1}(q)\left[\operatorname{Sat}_{\beta}(\hat{W}(t-T))-W_{d}(t)\right]
$$

and

$$
\begin{aligned}
N_{b} \triangleq \quad & {\left[M^{-1}(q)-M^{-1}\left(q_{d}\right)\right] M\left(q_{d}\right) \ddot{q}_{d} } \\
& +M^{-1}(q)\left[V_{m}\left(q_{d}, \dot{q}_{d}\right) \dot{q}_{d}-V_{m}(q, \dot{q}) \dot{q}\right. \\
& \left.+G\left(q_{d}\right)-G(q)+F_{d} \dot{e}\right] \\
& +M^{-1}(q)\left[k_{p} e+k_{c}(r+s)\right] \\
& +M^{-1}(q)\left[k_{L}(r+s)\right]
\end{aligned}
$$

We would like to note that applying (3), (6), (7), (10), (11), (12) and (13) to the auxiliary terms $N_{d}$ and $N_{b}$ can be bounded as follows

$$
\begin{aligned}
\left\|N_{d}\right\| & \leq \zeta_{N_{d}} \\
\left\|N_{b}\right\| & \leq \rho_{01}\|e\|+\rho_{02}\|r\|+\rho_{03}\|s\|+\rho_{04}\|r\|^{2}
\end{aligned}
$$

where $\zeta_{N_{d}}, \rho_{01}, \rho_{02}, \rho_{03}, \rho_{04} \in \mathbb{R}$ are positive known bounding constants.

\section{B. Error System Development}

To obtain the tracking error dynamics, we start with taking the time derivative of $r(t)$ in (18), then pre-multiply by $M(q)$, and utilize (1), (14), (23) to obtain

$$
M \dot{r}=-V_{m} r+\chi+W_{d}-\hat{W}-k_{p} e-k_{c}(r+s) .
$$

In (35), the auxiliary variable $\chi(t) \in \mathbb{R}^{n}$ is defined as

$$
\chi \triangleq M\left(\ddot{q}_{d}+\alpha \dot{e}\right)+V_{m}\left(\dot{q}_{d}+\alpha e\right)+G+F_{d} \dot{q}-W_{d} .
$$

Similar to the bound of (34), an upper bound for $\chi(t)$ can be obtained to have the following form

$$
\|\chi(t)\| \leq \rho_{1}(\|e\|)\|e\|+\rho_{2}(\|e\|)\|r\|
$$

where $\rho_{1}(\cdot), \rho_{2}(\cdot) \in \mathbb{R}$ are positive bounding functions that are in the following form

$$
\rho_{1}=\zeta_{1}+\zeta_{2}\|e\|, \rho_{2}=\zeta_{3}+\zeta_{4}\|e\|
$$

with $\zeta_{1}, \zeta_{2}, \zeta_{3}, \zeta_{4} \in \mathbb{R}$ being known positive bounding constants.

\section{Boundedness/Stability PRoOF}

The closed-loop error systems in (30) and (35) yields the following theorem to analyze the stability of observation error and position tracking error.

Theorem 1: The velocity observer in (20) and the control law in (23) ensures the closed-loop observer/controller couple is semi-globally asymptotically stable in the sense that

$$
\|e(t)\|,\|\dot{\tilde{q}}(t)\| \rightarrow 0 \text { as } t \rightarrow 0
$$

provided that the observer gain is selected to satisfy (29), the controller gain $k_{c}$ is designed as

$$
k_{c}=\left(k_{n} \zeta_{1}^{2}+k_{n} \zeta_{2}^{2}+\zeta_{3}+1\right) I_{n}
$$

and the observer gain $k_{2}$ is designed as

$$
k_{2}=\alpha\left(k_{n} \rho_{01}^{2}+k_{n} \rho_{02}^{2}+k_{n} \rho_{03}^{2}+\rho_{04}+k_{n} k_{L}^{2}+1\right) I_{n}
$$

where $k_{n}$ is a positive damping constant, and $\rho_{0 i}$ and $\zeta_{i}$ $i=1, \ldots, 4$ were defined in (34) and (38).

Proof: The proof is composed of four sub-proofs where only a highlight of the first part is given and the rest is presented in detail.

Firstly, the nonnegative function, denoted by $V_{1}(r, e, s) \in$ $\mathbb{R}$, is defined

$$
V_{1} \triangleq \frac{1}{2} r^{T} M r+\frac{1}{2} e^{T} k_{p} e+\frac{1}{2} s^{T} s .
$$

After taking the time derivative of $V_{1}(t)$ and substituting for (18), (30) and (35), results in

$$
\dot{V}_{1} \leq-\gamma_{0} V_{1}+\epsilon_{0}
$$

where $\gamma_{0}$ and $\epsilon_{0}$ are positive constants. From the structures of (42) and (43), it is easy to see that $V_{1}(t) \in \mathcal{L}_{\infty}$ and thus $e(t), r(t), s(t) \in \mathcal{L}_{\infty}$. Standard signal chasing arguments are then applied to demonstrate the boundedness of all the signals under the closed-loop operation, including $\tilde{q}(t)$ and $\dot{\tilde{q}}(t)$. 
Next, provided that $\tilde{q}(t)$ and $\dot{\tilde{q}}(t)$ are bounded, the following expression for the upper bound of the integral of the absolute value of the $i^{\text {th }}$ entry of $\dot{\tilde{q}}(t), i=1, \cdots, n$, can be obtained

$$
\int_{t_{0}}^{t}\left|\dot{\tilde{q}}_{i}(\sigma)\right| d \sigma \leq \gamma_{1}+\gamma_{2} \int_{t_{0}}^{t}\left|\tilde{q}_{i}(\sigma)\right| d \sigma+\left|\tilde{q}_{i}\right|
$$

where $\gamma_{1}, \gamma_{2} \in \mathbb{R}$ are some positive bounding constants. The proof of (44) can be found in [15] or in [16].

At this stage, to prove the overall stability of the closedloop system and the asymptotic convergence of the error signals, we define the following non-negative function, denoted by $V(t) \in \mathbb{R}$,

$$
\begin{aligned}
V & \triangleq V_{1}+P \\
& +\frac{1}{2 k_{L}} \int_{t-T}^{t}\left\|\operatorname{Sat}_{\beta}\left(W_{d}(\sigma)\right)-\operatorname{Sat}_{\beta}(\hat{W}(\sigma))\right\|^{2} d \sigma .
\end{aligned}
$$

where $P(t) \in \mathbb{R}$ is an auxiliary term defined as

$$
P \triangleq \zeta_{p}-\int_{0}^{t} s^{T}(\sigma)\left[N_{d}(\sigma)-k_{1} \operatorname{Sgn}(\tilde{q}(\sigma))\right] d \sigma
$$

where $\zeta_{p}$ is a positive constant. Provided the entries of the control gain matrix $k_{1}$ are chosen to be greater than the upper bound of the auxiliary term $N_{d}(t)$, the proof in [16] can be traced to demonstrate the non-negativeness of $P(t)$.

After taking time derivative of (45), then substituting (30), (35), (36), and performing some straightforward algebric manipulations, we obtain following expression

$$
\begin{aligned}
\dot{V}= & -r^{T} k_{c} r-\alpha e^{T} k_{p} e-\frac{1}{\alpha} s^{T} k_{2} s+r^{T} \chi+s^{T} N_{b} \\
& +\frac{1}{2 k_{L}}\left\|\operatorname{Sat}_{\beta}\left(W_{d}(t)\right)-\operatorname{Sat}_{\beta}(\hat{W}(t))\right\|^{2} \\
& -\frac{1}{2 k_{L}}\left\|\left[W_{d}-\hat{W}\right]\right\|^{2} \\
& -\frac{k_{L}}{2}\|(r+s)\|^{2}+k_{L} s^{T}(r+s) .
\end{aligned}
$$

The following relationship can be obtained for second and third lines of (47) as [7]

$$
\left\|\operatorname{Sat}_{\beta}\left(W_{d}(t)\right)-\operatorname{Sat}_{\beta}(\hat{W}(t))\right\|^{2}-\left\|\left[W_{d}-\hat{W}\right]\right\|^{2} \leq 0 .
$$

After utilizing (34), (37), and (48) along with (47), and then applying the nonlinear damping argument to the resulting expression, we can obtain

$$
\dot{V} \leq-\left[\min \{\alpha, 1\}-\frac{3}{k_{n}}-\frac{1}{k_{n}}\|z\|^{2}\right]\|z\|^{2} .
$$

Where $z=[e, r, s]^{T}$ denotes combined error signals. It is now possible to upper bound the right-hand side of (49) as

$$
\dot{V} \leq-\gamma_{4}\|z\|^{2}
$$

where $\gamma_{4} \in \mathbb{R}$ is some positive constant $\left(0<\gamma_{4} \leq 1\right)$. Barbalat's Lemma [13], [14] can now be utilized to prove the semi-global asymptotic convergence of the joint velocity estimation error and the tracking error to the origin.

\section{Simulation Results}

To illustrate the performance of the observer based output feedback controller in (23), we performed a numerical simulation with a two link, planar robot manipulator. The dynamic model in (1) is considered with the following model functions

$$
\begin{aligned}
M & =\left[\begin{array}{cc}
p_{1} & -0.5 p_{2} s_{\left(q_{1}-q_{2}\right)} \\
-0.5 p_{2} s_{\left(q_{1}-q_{2}\right)} & p_{3}
\end{array}\right] \\
V_{m} & =\left[\begin{array}{cc}
0 & 0.5 p_{2} \dot{q}_{2} c_{\left(q_{1}-q_{2}\right)} \\
-0.5 p_{2} \dot{q_{1}} c_{\left(q_{1}-q_{2}\right)} & 0
\end{array}\right] \\
N & =\left[\begin{array}{c}
p_{4} c_{q_{1}}+p_{5} c_{\left(q_{1}-0.5 \pi\right)} \\
p_{6} s_{q_{2}}
\end{array}\right]
\end{aligned}
$$

in which $s_{q_{2}}=\sin \left(q_{2}\right), c_{q_{2}}=\cos \left(q_{2}\right), s_{\left(q_{1}-q_{2}\right)}=\sin \left(q_{1}-\right.$ $\left.q_{2}\right), c_{\left(q_{1}-q_{2}\right)}=\cos \left(q_{1}-q_{2}\right), c_{\left(q_{1}-0.5 \pi\right)}=\cos \left(q_{1}-0.5 \pi\right)$ and $p_{1}=2.52610^{-3}, p_{2}=2.76610^{-3}, p_{3}=1.65210^{-3}$, $p_{4}=164.15810^{-3}, p_{5}=117.29410^{-3}$ and $p_{6}=94.0510^{-3}$. We would like to note that the above dynamic model is not utilized in the control design when performing the numerical simulations.

The periodic desired joint space trajectory was selected as

$$
q_{d}=\left[\begin{array}{c}
(0.8+0.2 \sin (0.5 t)) \sin (0.5 \sin (0.5 t)) \\
(0.6+0.2 \sin (0.5 t)) \sin (0.5 \sin (0.5 t))
\end{array}\right] .
$$

The robot manipulator is considered to be at rest with the initial joint position as $q(0)=[0.1,0.1]^{T}$ rad. Satisfactory tracking performance is obtained when the gains set on, $k_{p}=$ $[0.1,0 ; 0,0.1], k_{c}=[0.08,0 ; 0,0.08], k_{0}=[500,0 ; 0,500]$, $k_{1}=[0.1,0 ; 0,0.1], \alpha=5.2, k_{2}=\alpha\left(k_{0}-\alpha\right)$ and $k_{L}=0.1$.

The joint space tracking error $e(t)$ is shown in Figure 1 . Auxiliary possition observation error $\tilde{q}$ is shown in Figure 2 . Control input torque can be seen in Figure 3. The desired and the actual joint space trajectories can be seen from Figure 4. From Figures 1, 2 and 4, it is clear that the tracking objective was successfully met. Specifically, from Figure 1, it is clearly observed that the proposed learning controller ensures a significant improvement on the tracking error in every period (which was $12.5 \mathrm{sec}$ ).

\section{CONCLUSION}

In this study, we have presented an observer based output feedback learning controller for tracking control of robot manipulators. A novel observer-controller formulation that ensured semi-globally asymptotic tracking despite the lack of link velocity measurements have been presented. The convergence of the error signals and the stability of the closed-loop system are illustrated via Lyapunov type analysis. Numerical solutions are demonstrated to illustrate the tracking performance of the proposed method.

There is much to be considered as future work. One future work is to obtain experimental verification of the proposed controller-observer couple on a robotic platform. Another possible interesting future research is applying the proposed control method to telerobotic systems when the desired task has a repeating structure. Another branch of future work is to apply the proposed technique to control of active magnetic bearings [17] and atomic force microscopy [18]. While this 

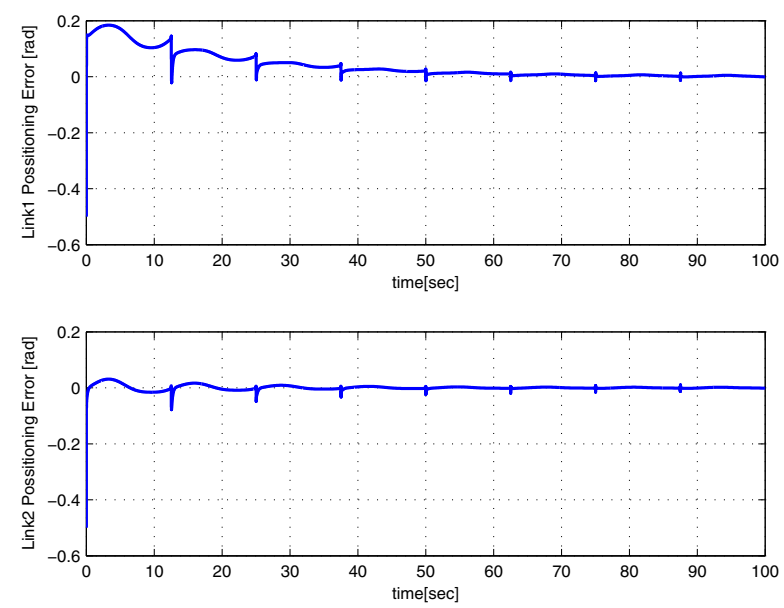

Fig. 1. Joint position tracking error $e(t)$

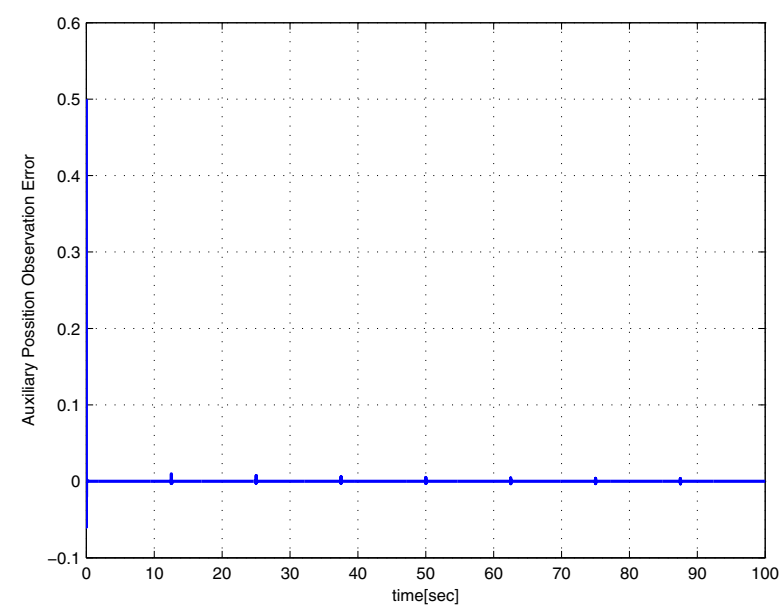

Fig. 2. Auxiliary possition observation error $\tilde{q}$

work is presented for robot manipulators, it is possible to apply the proposed methodology to other Euler-Lagrange systems mentioned above. While the control problem in the above mentioned applications are rejecting a periodic disturbance type effects (rather than following a periodic reference trajectory), the proposed method can with some effort be applied to address those research problems.

\section{APPENDIX I}

\section{PROOF OF BOUNDS}

In this appendix, we discuss how the upper bound for $N_{b}(t)$ in (34) can be obtained. Obtaining the upper bound for $\chi(t)$ in (37) is possible after following similar steps.

Starting with rewriting the expression (32) and utilizing
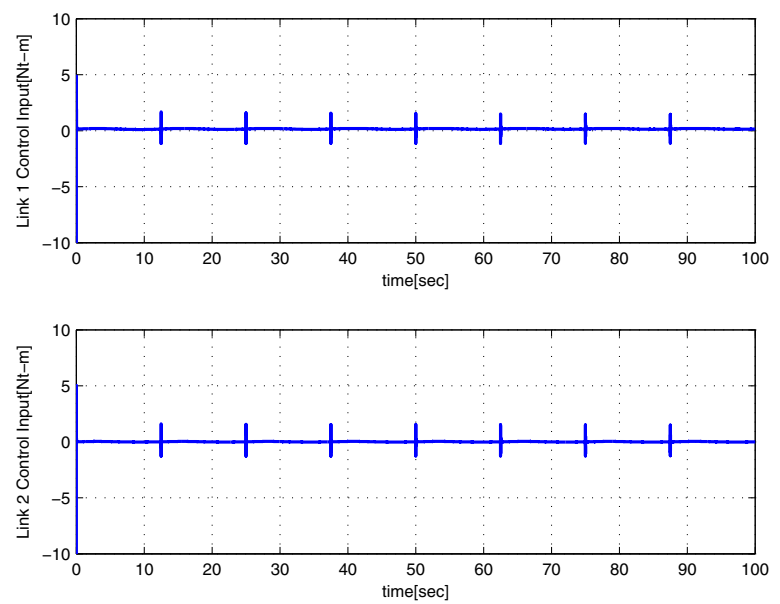

Fig. 3. Control torque input $\tau(t)$
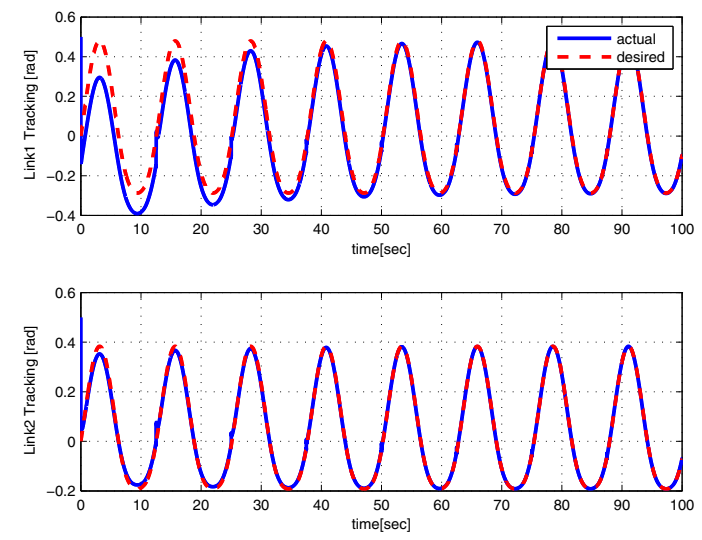

Fig. 4. Desired (dashed) and actual joint space trajectories

(5) we will obtain

$$
\begin{aligned}
N_{b} & =\left(M^{-1}(q)-M^{-1}\left(q_{d}\right)\right) M^{-1}\left(q_{d}\right) \ddot{q}_{d} \\
& +M^{-1}(q)\left\{V_{m}\left(q_{d}, \dot{q}_{d}\right) \dot{q}_{d}-V_{m}\left(q, \dot{q}_{d}\right) \dot{q}_{d}\right\} \\
& +M^{-1}(q)\left\{2 V_{m}(q, \dot{e}) \dot{q}_{d}-V_{m}(q, \dot{e}) \dot{e}\right\} \\
& +M^{-1}(q)\left\{G\left(q_{d}\right)-G(q)+F_{d}\left(\dot{q}_{d}-\dot{q}\right)\right\} \\
& +M^{-1}(q)\left\{k_{p} e+k_{c} r+k_{c} s\right\} .
\end{aligned}
$$

After utilizing (3), (6), (7), (10), (11), (12) and (13) we can obtain an upper bound for the right-hand-side of (55) as follows

$$
\begin{aligned}
N_{b} & \leq\left\{\zeta_{M 1} m_{2}\left\|\ddot{q}_{d}\right\|+\frac{1}{m_{1}} \zeta_{c 2}\left\|\dot{q}_{d}\right\|\right\}\|e\| \\
& +\left\{\frac{1}{m_{1}} \lambda_{\max } k_{p}+\frac{1}{m_{1}} \zeta_{g}\right\}\|e\| \\
& +\left\{\frac{2}{m_{1}} \zeta_{c 1}\left\|\dot{q}_{d}\right\|+\frac{1}{m_{1}} \zeta_{f}+\frac{1}{m_{1}} \lambda_{\max } k_{c}\right\}\|r\| \\
& +\frac{1}{m_{1}} \zeta_{c 1}\|r\|^{2}+\frac{1}{m_{1}} \lambda_{\max } k_{c}\|s\|
\end{aligned}
$$


where the fact that $\|r(t)\| \geq\|\dot{e}(t)\|$ is utilized. Let

$$
\begin{aligned}
\rho_{01} & \triangleq \zeta_{M 1} m_{2}\left\|\ddot{q}_{d}\right\|+\frac{1}{m_{1}} \zeta_{c 2}\left\|\dot{q}_{d}\right\| \\
& +\frac{1}{m_{1}} \lambda_{\max } k_{p}+\frac{1}{m_{1}} \zeta_{g} \\
\rho_{02} & \triangleq \frac{2}{m_{1}} \zeta_{c 1}\left\|\dot{q}_{d}\right\|+\frac{1}{m_{1}} \zeta_{f}+\frac{1}{m_{1}} \lambda_{\max } k_{c} \\
\rho_{03} & \triangleq \frac{1}{m_{1}} \zeta_{c 1} \\
\rho_{04} & \triangleq \frac{1}{m_{1}} \lambda_{\max } k_{c}
\end{aligned}
$$

then the bound given in (34) is valid.

\section{REFERENCES}

[1] S. Hara, Y. Yamamoto, T. Omata, and M. Nakano, "Repetitive control systems: A new type servo system for periodic exogenous signals," IEEE Transactions on Robotics, vol. 33, no. 7, pp. 659-668, 1988.

[2] M. Tomizuka, T. Tsao, and K. Chew, "Discrete-time domain analysis and synthesis of repetitive controllers," ASME Journal on Dynamic Systems, Measurement, and Control, vol. 111, pp. 353-358, 1989.

[3] M. Tsai, G. Anwar, and M. Tomizuka, "Discrete-time repetitive control for robot manipulators," in Proc. IEEE Int. Conf. Robot. Autom., Philadelphia, PA, 1988, pp. 1341-1347.

[4] W. Messner, R. Horowitz, W. Kao, and M. Boals, "A new adaptive learning rule," IEEE Tr. on Autom. Contr., vol. 36, pp. 188-197, 1991.

[5] R. Horowitz, "Learning control of robot manipulators," ASME J. Dyna. Syst., Meas. Contr., vol. 115, pp. 402-411, 1993.

[6] N. Sadegh and R. Horowitz, "Stability and robustness analysis of a class of adaptive controllers for robot manipulators," Int. J. Robotics Research, vol. 9, no. 3, pp. 74-92, 1990.

[7] W. E. Dixon, E. Zergeroglu, D. M. Dawson, and B. T. Costic, "Repetitive learning control : A Lyapunov-based approach," IEEE Tr. on Systems, Man, and Cybernetics, vol. 32, no. 4, pp. 538-545, 2002.

[8] P. He and S. Jagannathan, "Reinforcement learning based outputfeedback control of nonlinear systems with input constraints," in Proc. of the 2004 American Control Conf., Boston, MA- USA, 2004, pp. 2563-2568.

[9] P. Shih and S. Jagannathan, "Reinforcement learning based outputfeedback controller for complex nonlinear discrete-time systems," in 22nd IEEE Int. Symp. on Int. Control Part of IEEE Multi-Conference on Syst. and Control, Suntec City, Singapore, 2007, pp. 407-412.

[10] R. Marino, P. Tomei, and C. Verrelli, "Learning control for nonlinear systems in output feedback form," Systems \& Control Letters, vol. 61, pp. 1242-1247, 2012.

[11] D. M. Dawson, M. M. Bridges, and Z. Qu, Nonlinear Control of Robotic Systems for Environmental Waste and Restoration. Englewood Cliffs, NJ, USA: Prentice Hall, 1995.

[12] J. Nakanishi, R. Cory, M. M. J. Peters, and S. Schaal, "Operational space control: A theoretical and emprical comparsion," Int. J. Robotics Research, vol. 27, no. 6, pp. 737-757, 2008.

[13] F. L. Lewis, D. M. Dawson, and C. T. Abdallah, Robot Manipulator Control: Theory and Practice. New York, NY, USA: Marcel Dekker, Inc., 2003.

[14] F. L. Lewis, C. T. Abdallah, and D. M. Dawson, Control of Robot Manipulators. New York, NY, USA: MacMillan, 1993.

[15] V. Stepanyan and A. Kurdila, "Asymptotic tracking of uncertain systems with continuous control using adaptive bounding," IEEE Trans. Neural Netw., vol. 20, no. 8, pp. 1320-1329, 2009.

[16] B. Bidikli, E. Tatlicioglu, E. Zergeroglu, and A. Bayrak, "An asymptotically stable continuous robust controller for a class of uncertain MIMO nonlinear systems," ArXiv e-prints, Tech. Rep. 1301.5483, Jan. 2013.

[17] B. T. Costic, M. S. deQueiroz, and D. M. Dawson, "A new learning control approach to the active magnetic bearing benchmark system," in Proc. American Control Conf., Chicago, IL, USA, 2000, pp. 59895994.

[18] Y. Fang, M. G. Feemster, D. M. Dawson, and N. Jalili, "Nonlinear control techniques for the atomic force microscope system," in ASME Int. Mechanical Eng. Congress, New Orleans, LA, USA, 2002, pp. $1-8$. 